Bound electron pairs in strongly correlated models of high-temperature superconductivity

H. Boyaci, and I. O. Kulik

Citation: Low Temperature Physics 24, 239 (1998);

View online: https://doi.org/10.1063/1.593577

View Table of Contents: http://aip.scitation.org/toc/ltp/24/4

Published by the American Institute of Physics

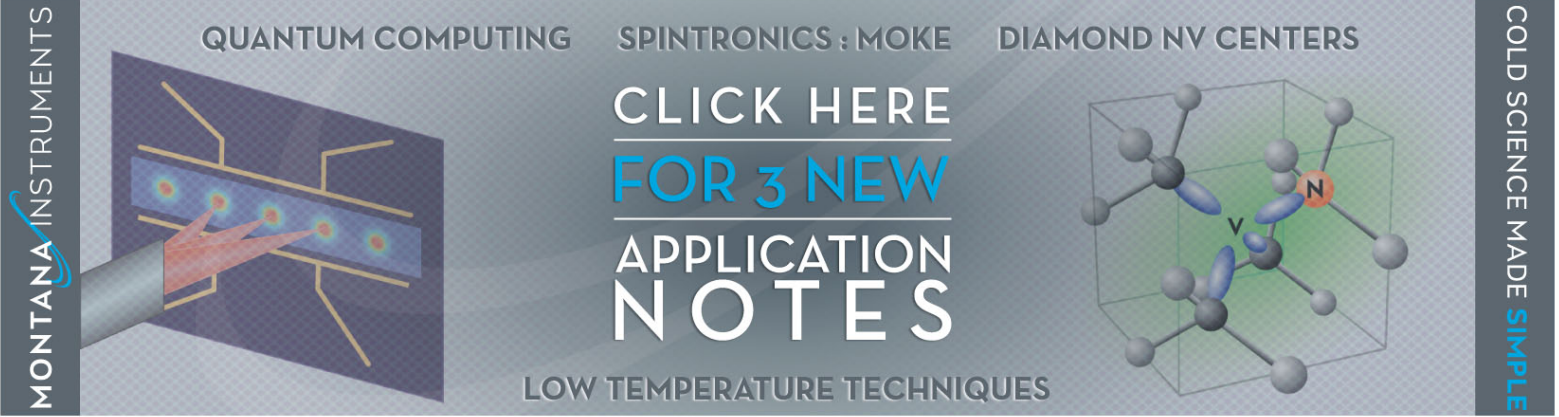




\title{
Bound electron pairs in strongly correlated models of high-temperature superconductivity
}

\author{
H. Boyaci and I. O. Kulik*
}

Department of Physics, Bilkent University, Bilkent, 06533 Ankara, Turkey**

(Submitted August 7, 1997; revised November 17, 1997)

Fiz. Nizk. Temp. 24, 316-325 (April 1998)

The ground-state energy of two electrons on a ring is calculated for the one-dimensional Hubbard model with positive and negative on-site interaction and for the contraction model with additive and multiplicative interaction terms. The $h c / 2 e$ periodicity of the ground-state energy with respect to a flux $\Phi$ threading the loop is derived. The periodicity may serve as an indication of superconductivity. The results are shown to be consistent with the Lieb-Wu solution for $\Phi=0$ limit. In addition, the new states that were missing in the Lieb-Wu solution are derived. (C) 1998 American Institute of Physics. [S1063-777X(98)00504-0]

\section{INTRODUCTION}

Among the possible mechanisms of high temperature superconductivity attention was focused in the last years on strongly correlated systems, ${ }^{1}$ non Fermi-liquid scenarios, ${ }^{2,3}$ magnetic schemes (spin-fluctuation ${ }^{4,5}$ and spin-bag ${ }^{6}$ ) and soft orbital mode interaction mechanisms. ${ }^{7,8}$ The generic Hamiltonian underlying these models are the one-, two-, or threeband Hubbard positive- or negative- $U$ Hamiltonians and contraction Hamiltonians with a hopping amplitude which depends upon the sum or product of the near-site occupation number operators. The criterion for superconductivity can be learned in the pairing instability, in the Meissner effect, or in flux quantization. In this paper some of the above models are considered in an assumption that halving of the flux periodicity in the energy versus flux dependence ( $h c / e$ to $h c / 2 e$ ) may serve as an indication of the superconducting transition.

The purpose of this paper is to show some new states for the one-dimensional Hubbard model, which are missing in the Lieb- $\mathrm{Wu}^{9}$ solution, and to show that the contraction model may serve as a mechanism for superconductivity. Similar states appear in other strongly correlated models of high- $T_{c}$ superconductivity. Specifically, we will analyze in this paper three Hamiltonians for strongly correlated fermions:

(1) Hubbard model with repulsive on-site interaction. ${ }^{5}$

(2) Negative- $U$ Hubbard Hamiltonians. ${ }^{27,28}$

(3) Contraction-pairing mechanisms. ${ }^{7,8,10}$

It is known that direct O-O hopping in high- $T_{c}$ superconductors is important. Since oxygen in oxides like $\mathrm{YBa}_{2} \mathrm{Cu}_{3} \mathrm{O}_{6+x}$ has almost filled $p$-shell configuration, holes in a $p^{6}$ shell may play a similar role for the conduction in oxides in question, as the electrons from nearly empty atomic shells in conventional metals do. Oxygen atoms are specific in the sense that change of the oxygen ionization state $\left(\mathrm{O}^{0}\right.$ to $\mathrm{O}^{-}$and $\left.\mathrm{O}^{2-}\right)$ results in a dramatic increase of $p_{x}, p_{y}$ orbitals in the $\mathrm{CuO}$ plane, and therefore in the increase of the magnitude of hopping between near oxygen (as well as near oxygen-copper) sites. A non-s-wave orbital configuration ${ }^{10}$ is expected to survive with consideration of this occupation-dependent hopping.

\section{GROUND-STATE ENERGY OF TWO ELECTRONS IN THE HUBBARD MODEL WITH POSITIVE AND NEGATIVE ON-SITE INTERACTION}

We consider a loop of $N_{a}$ lattice sites with a magnetic flux $\Phi$ threading the loop (Fig. 1). The electrons can hop between neighboring lattice sites, and each site can be occupied by at most two electrons with opposite spins. The Hamiltonian for this system has the form

$$
\begin{aligned}
H= & -t \sum_{j, \sigma}\left(c_{j, \sigma}^{+} c_{j+1, \sigma} \mathrm{e}^{i \alpha}+c_{j+1, \sigma}^{+} c_{j, \sigma} \mathrm{e}^{-i \alpha}\right) \\
& +U \sum_{j} n_{j \uparrow} n_{j \downarrow},
\end{aligned}
$$

where $c_{j, \sigma}^{+}$and $c_{j \sigma}$ are respectively the creation and annihilation operators of an electron with spin projection $\sigma$ at the $j$ th lattice site, $t$ is the electron hopping amplitude, $\alpha=\left(2 \pi / N_{a}\right)\left(\Phi / \Phi_{0}\right)$ (here $\Phi_{0}=h c / e$ is the magnetic flux quantum), $n_{j \sigma}$ is the occupation number operator, and $U$ is on-site interaction term. The energy spectrum of $H$ is invariant under the replacement of $t$ by $-t$. Hence, we assume $t=+1$ in appropriate units.

The wave function for two electrons, one with spin up and the other with spin down, is

$$
|\Psi\rangle=\sum_{x_{1}, x_{2}} f\left(x_{1}, x_{2}\right) c_{x_{1} \downarrow}^{+} c_{x_{2} \uparrow}^{+}|0\rangle,
$$

where $|0\rangle$ is a vacuum state.

The eigenvalue equation $H|\Psi\rangle=E|\Psi\rangle$ leads to

$$
\begin{aligned}
& -\left[\left(f\left(x_{1}+1, x_{2}\right)+f\left(x_{1}, x_{2}+1\right)\right) \mathrm{e}^{i \alpha}+\left(f\left(x_{1}-1, x_{2}\right)\right.\right. \\
& \left.\left.\quad+f\left(x_{1}, x_{2}-1\right)\right) \mathrm{e}^{-i \alpha}\right]+U \delta\left(x_{1}, x_{2}\right) f\left(x_{1}, x_{2}\right) \\
& =E f\left(x_{1}, x_{2}\right)
\end{aligned}
$$

or, in the momentum space, 


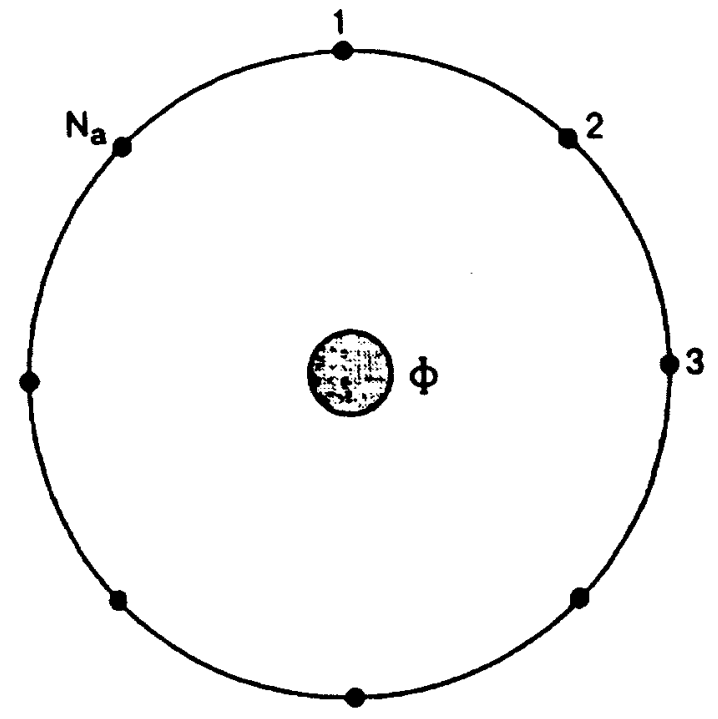

FIG. 1. Configuration of the sample. There are $N_{a}$ lattice sites on the ring which can be numbered from 1 to $N_{a}$. The flux $\Phi$ piercing the ring is produced by a solenoid inserted inside the ring.

$$
\begin{aligned}
(E & \left.+2 \cos \left(K_{1}+\alpha\right)+2 \cos \left(K_{2}+\alpha\right)\right) f_{K_{1}, K_{2}} \\
& =\frac{U}{N_{a}} \sum_{K} f_{K_{1}-K, K_{2}+K},
\end{aligned}
$$

where $K_{1,2}=\left(2 \pi / N_{a}\right) n_{1,2}$ with $n_{1,2}=0,1,2, \ldots, N_{a}-1$. Here $f_{K_{1}, K_{2}}$ is assumed to satisfy the periodicity condition $f_{K_{1}+2 \pi, K_{2}}=f_{K_{1}, K_{2}+2 \pi}=f_{K_{1}, K_{2}}$. Equation (4) can be rewritten as follows:

$$
\begin{aligned}
& P_{Q}\left(1-\frac{U}{N_{a}}\right. \\
& \left.\quad \times \sum_{p} \frac{1}{E+2 \cos \left(K_{1}-p+\alpha\right)+2 \cos \left(K_{2}+p+\alpha\right)}\right)=0,
\end{aligned}
$$

where $P_{Q}=\left(1 / N_{a}\right) \Sigma_{K} f_{K_{1}-K, K_{2}+K}, \quad Q=K_{1}+K_{2}=(2 \pi /$ $\left.N_{a}\right) n$, and $p=\left(2 \pi / N_{a}\right) m$. Hence, either the term inside the parentheses or $P_{Q}$ should be equal to zero.

(I) $P_{Q} \neq 0$. The Lieb and $W u$ solution

For $P_{Q} \neq 0$, the term inside the parentheses should be equal to zero, or

$$
\frac{1}{U}=S(E)
$$

where

$$
S(E)=\frac{1}{N_{a}} \sum_{p} \frac{1}{E+2 \cos \left(K_{1}-p+\alpha\right)+2 \cos \left(K_{2}+p+\alpha\right)} .
$$

Using the Poisson summation formula,

$$
\frac{1}{U}=\sum_{n=-\infty}^{\infty} \int_{0}^{2 \pi} \frac{d p}{2 \pi} \frac{\exp \left(i p N_{a} n\right)}{E+4 \cos (Q / 2-p) \cos (Q / 2+\alpha)}
$$

$S(E)$ becomes
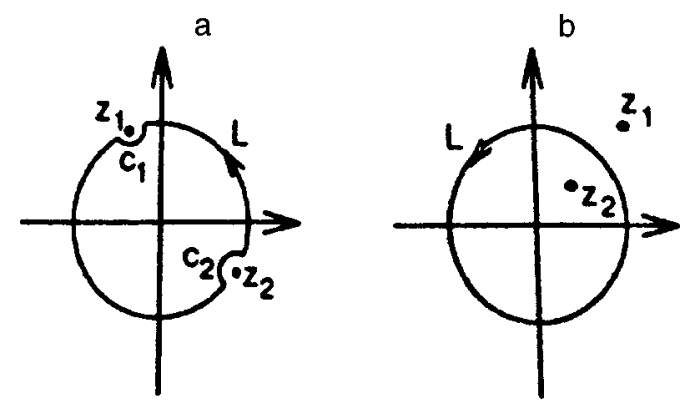

FIG. 2. Poles of the integrand in the complex plane. $E^{2}<E_{0}^{2}$ (a) and $E^{2}>E_{0}^{2}(\mathrm{~b})$, where $E_{0}=-4 \cos \beta$ for even $n$ and $E_{0}=-4 \cos \beta \cos \left(\pi / N_{a}\right)$ for odd $n$.

$$
S(E)=\sum_{n=-\infty}^{\infty} S_{n}(E) \equiv S_{n=0}(E)+\sum_{n=1}^{\infty}\left[S_{n}(E)+S_{n}^{*}(E)\right]
$$

$S_{n}(E)$ can be calculated by transforming Eq. (8) to an integral in the complex plane. Setting $z=\mathrm{e}^{i p}$, we have

$$
\begin{aligned}
S_{n}(E) & =\frac{1}{2 \pi i} \\
& \times \oint d z \frac{z^{N_{a} n}}{z^{2}\left(\mathrm{e}^{i \alpha}+\mathrm{e}^{-i(Q+\alpha)}\right)+E z+\left(\mathrm{e}^{i(Q+\alpha)}+\mathrm{e}^{-i \alpha}\right)} .
\end{aligned}
$$

The poles (Fig. 2) of the integrand are

$$
z_{1,2}=-\frac{-E \pm\left(E^{2}-E_{0}^{2}\right)^{1 / 2}}{E_{0} \exp (-i Q / 2)}
$$

where $E_{0}=4 \cos (Q / 2+\alpha)$. For $E^{2}<E_{0}^{2}$, both of the poles $z_{1}$ and $z_{2}$ are on the unit circle and $S_{n=0}$ vanishes, while for $E^{2}>E_{0}^{2}$ one of them is inside the unit circle and the other one is outside of it, and $S_{n=0}$ does not vanish. For both cases

$$
S(E)=\frac{1}{4 i \sin x \cos \beta} \frac{\exp \left(i(Q / 2-x) N_{a}\right)+1}{\exp \left(i(Q / 2-x) N_{a}\right)-1},
$$

where $x$ can be real or complex, depending on whether $E^{2}$ is smaller or larger than $E_{0}^{2}$, and $\beta=Q / 2+\alpha$. If we denote new momenta $k_{1}, k_{2}$ as

$$
k_{1,2}=\frac{Q}{2}+\alpha \pm x,
$$

Eq. (6) takes the form

$$
\exp \left[i\left(k_{1,2}-\alpha\right) N_{a}\right]=\frac{\sin k_{1,2}-\Lambda+i U / 4}{\sin k_{1,2}-\Lambda-i U / 4},
$$

where

$$
\Lambda=\frac{\sin k_{1}+\sin k_{2}}{2} .
$$

Equation (14) is identical to the Lieb and $\mathrm{Wu}$ solution ${ }^{9}$ in the $\alpha=0$ limit.

It is possible to express the eigenvalue $E$ of the system as 
TABLE I. Minimum energy for different values of $U$.

\begin{tabular}{|c|c|c|c|}
\hline & $U>0$ & \multicolumn{2}{|c|}{$U<0$} \\
\hline \multirow[t]{2}{*}{ even $n$} & $\begin{array}{l}E=-4 \cos x \cos \beta \\
\text { with } x \text { (real) determined by } \\
\tan \left(N_{a} x / 2\right)=U / 4 \sin x \cos \beta\end{array}$ & \multicolumn{2}{|c|}{$\begin{array}{c}E=-4 \cosh \kappa \cos \beta \\
\text { with } \kappa \text { determined by } \\
\tanh \left(N_{a} \kappa / 2\right)=|U| / 4 \sinh \kappa \cos \beta\end{array}$} \\
\hline & & $U<U_{\mathrm{cr}}$ & $U_{\mathrm{cr}}<U<0$ \\
\hline odd $n$ & $E=-4 \cos \beta \cos \left(\pi / N_{a}\right)$ & $\begin{array}{c}E=-4 \cosh \kappa \cos \beta \\
\text { where } \kappa \text { is determined by } \\
\tanh \left(N_{a} \kappa / 2\right)=4 \sinh \kappa \cos \beta /|U|\end{array}$ & $\begin{array}{c}E=-4 \cos x \cos \beta \\
\text { where } x \text { is determined by } \\
\tan \left(N_{a} \kappa / 2\right)=4 \sinh x \cos \beta /|U|\end{array}$ \\
\hline
\end{tabular}

$$
E=-2\left(\cos k_{1}+\cos k_{2}\right)=-4 \cos x \cos \beta,
$$

with $x$ determined by

$$
\tan \frac{N_{a} x}{2}=-\sigma\left(\frac{4 \sin x \cos \beta}{U}\right)^{\sigma},
$$

where $\sigma=+1$ or -1 for odd or even value of $n[n$ $\left.=Q /\left(2 \pi / N_{a}\right)\right]$.

For $U>0, E^{2}$ is always less than $E_{0}^{2}$; hence $x$ is always real. For $U<0$ with even $n, E^{2}$ is always larger than $E_{0}^{2}$, so that $x$ is complex. But for odd $n$ and small $|U|$ values ( $U$ $<0$ ), $x$ might be real. Let us consider Eq. (17) for negative $U$ and odd $n$ with complex $x=i \kappa$

$$
\frac{1}{|U|}=\frac{\tanh \left(N_{a} \kappa / 2\right)}{4 \sinh \kappa \cos \beta} \text {. }
$$

To have a solution of this equation, $1 /|U|$ should not be larger than the maximum value of its right-hand side. Accordingly, the critical value $\left|U_{\mathrm{cr}}\left(N_{a}\right)\right|$ can be found. The values of $|U|$ which are smaller than this $\left|U_{\text {cr }}\right|$ have real $x$; others have complex $x$ in Eq. (17).

\section{(II) $P_{Q}=0$. The new state}

If $P_{Q}$ is equal to zero, then either a new eigenvalue of the system is found as

$$
E=-2 \cos (q+\alpha)-2 \cos (Q-q+\alpha),
$$

with $K_{1}=q$ and $K_{2}=Q-q$, or $f_{K_{1} K_{2}}=0$ for any $K_{1}$ and $K_{2}$. But all $f$ 's cannot be zero; otherwise $|\Psi\rangle=0$. Summation of all $f$ 's, so that $P_{Q}$ is equal to zero while $f$ 's are individually not all zero only if for two different values of $q, 2 \cos (q$ $+\alpha)+2 \cos (Q-q+\alpha)$ are coinciding.

For positive on-site interaction $U$, this eigenvalue becomes the minimum energy of the system when $n$ is odd. For $U<0$ it does not become the minimum eigenvalue of the system.

The ground-state energy values are summarized in Table I.

The dependence of the ground-state energy on the flux is shown in Fig. 3.

\section{A. Dependence of the amplitude of energy oscillations on the number of sites}

The dependence $E(\Phi)$ is shown schematically in Fig. 4, where $\Delta E_{1}$ and $\Delta E_{2}$ are the amplitudes of $h c / e$ and $h c / 2 e$ oscillations.

For $U<U_{\mathrm{cr}}<0$ in the large $N_{a}$ limit

$$
\Delta E_{1}=\Delta E_{2}=\Delta E \approx \frac{2 \pi^{2}}{N_{a}^{2}} \frac{1}{\left(U^{2}+16\right)^{1 / 2}} .
$$

Here there is a $\Phi_{0} / 2$ periodicity, which resembles the pairing of electrons as in a superconductor, but the amplitude of the energy oscillations decreases with inverse square of the a

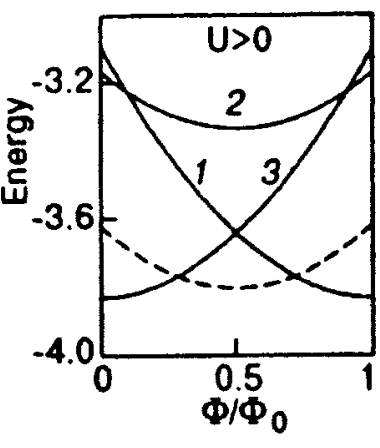

C

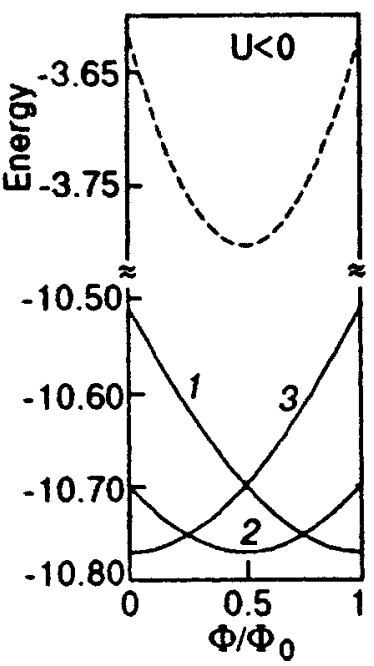

b

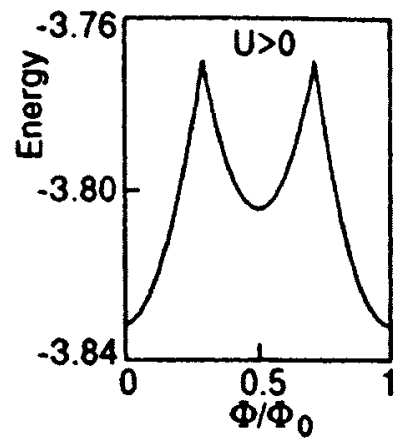

d

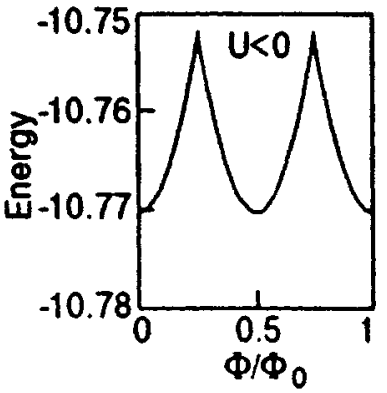

FIG. 3. Energy versus flux for two electrons with $N_{a}=10$. (a) Solid curves 1-3 correspond to the Lieb-Wu solution and the dashed curve corresponds to the new states found by us. For $U>0(U=10)$ this new state becomes the minimum energy of the system. (b) The same as (a) to show the $\Phi_{0} / 2$ periodicity more clearly. It is clearly seen that the Lieb-Wu solution (solid curves $1-3$ ) does not lead to the $\Phi_{0} / 2$ periodicity alone. (c) $U=-10$. As in (a), the solid curves $1-3$ are the lowest-lying eigenvalues found by the Lieb-Wu solution. Similarly, the dashed curve corresponds to the new state found by us. For $U<0$ the new eigenvalue does not become the minimum energy of the system. (d) The same as (c) to show the periodicity more clearly. 


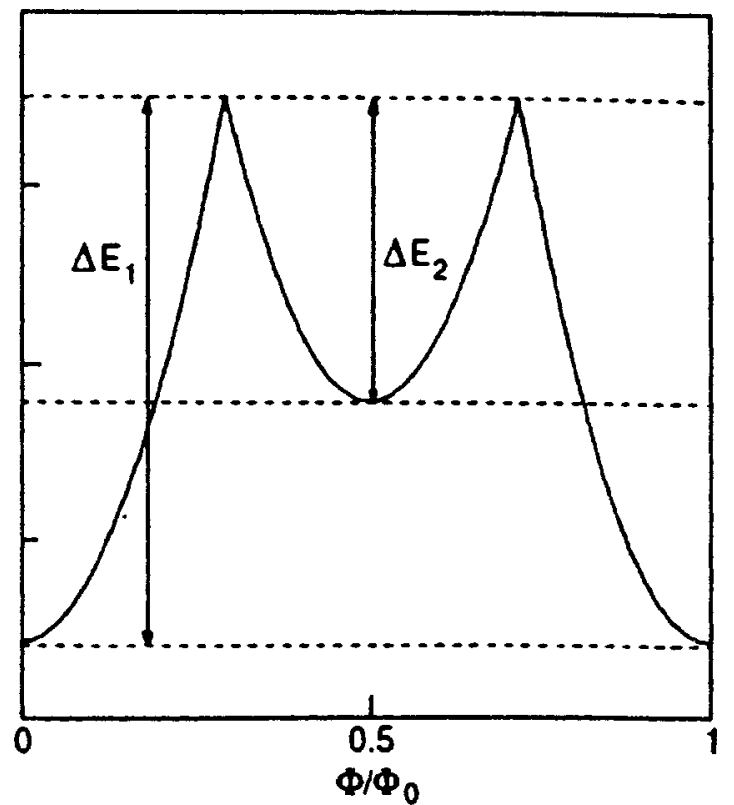

FIG. 4. Energy oscillations for two electrons. $\Delta E_{1}$-amplitude of hc/e periodicity, $\Delta E_{2}$-amplitude of $h c / 2 e$ periodicity.
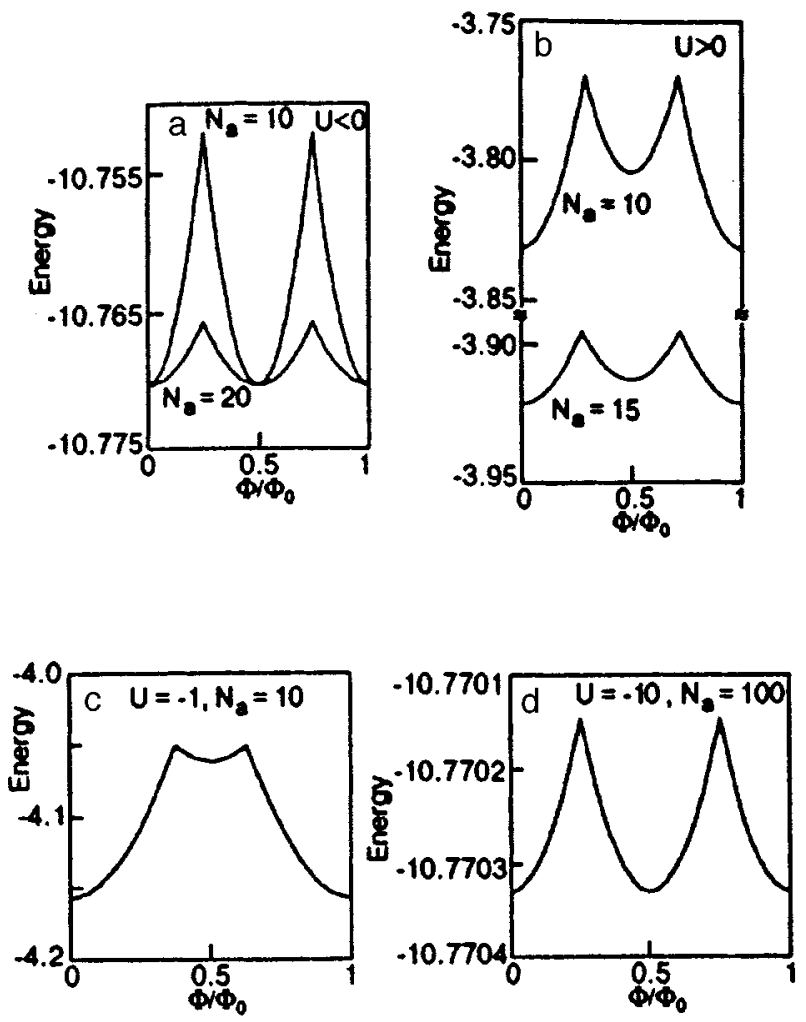

number of lattice sites (Fig. 5a). If $|U| \rightarrow\left|U_{\mathrm{cr}}\right|$, then the amplitude of oscillation corresponding to $h c / 2 e$ becomes smaller and at $U=U_{\mathrm{cr}}$ it vanishes. Note, however, that for very large values of $N_{a},\left|U_{\text {cr }}\right|$ becomes quite small; hence even for very small $|U|$ the behavior of energy with respect to flux is the same. The behavior of ground-state energy is shown explicitly for various values of $U$ and $N_{a}$ in Figs. 5c-5f. In the very large $N_{a}$ limit, using Eqs. (16) and (17), we can show that

$$
E \approx-\sqrt{U^{2}+16 \cos ^{2} \beta}
$$

for even and odd values of $n$. The last expression can be obtained directly from Eq. (7) by changing the summation over $p$ to an integral.

For $U>0$, in the limit $N_{a} \gg 1$

$$
\begin{aligned}
& \Delta E_{1} \approx \frac{2 \pi^{2}}{N_{a}^{2}}\left(1-\frac{1}{2}\left(\frac{U N_{a}}{8+U N_{a}}\right)^{2}\right)^{2}, \\
& \Delta E_{2} \approx \frac{2 \pi^{2}}{N_{a}^{2}}\left(\frac{1}{2}\left(\frac{U N_{a}}{8+U N_{a}}\right)^{2}\right)^{2} .
\end{aligned}
$$

Hence, for $U \times N_{a} \rightarrow \infty, \Delta E_{1}=\Delta E_{2}=1 / 4\left(2 \pi^{2} / N_{a}^{2}\right)$. Both $\Delta E_{1}$ and $\Delta E_{2}$ behave like $1 / N_{a}^{2}$, and $\Delta E_{1} / \Delta E_{2} \rightarrow 1$ (Fig.
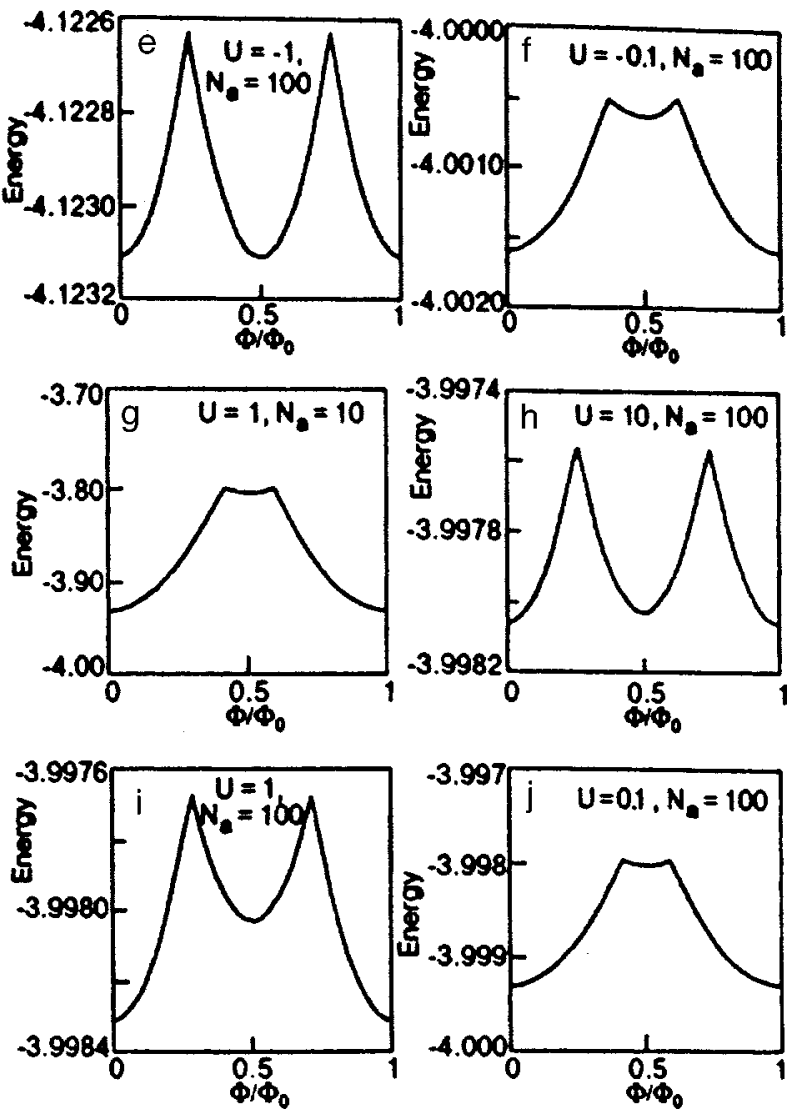

FIG. 5. (a) Minimal energy versus flux for $N_{a}=10$ and $20(U=-10)$. Comparison of oscillations for $N_{a}=10$ and 20 shows the $1 / N_{a}^{2}$ behavior of the amplitude. (b) Minimal energy versus flux for $N_{a}=10$ and $15(U=10)$. As the number of sites increases (larger $\left.N_{a}\right), \Delta E_{1} / \Delta E_{2}$ approaches 1 . (c-f) Ground-state energy for different values of $N_{a}$ and negative $U$. Compared to the oscillations in (a) for $N_{a}=10$ amplitude $\Delta E_{2}$ becomes smaller in (c). This occurs because $U$ comes closer to $U_{\text {cr }}$, if larger values of $U$ were used, even smaller $\Delta E_{2}$ values would be obtained. (d), (e), and (f) demonstrates the behavior of the system with $N_{a}=100$. This time even with $U=-1, \Delta E_{2}$ is still almost equal to $\Delta E_{1}$, because for larger values of $N_{a}, U_{\text {cr }}$ becomes larger and approaches zero. For $U=-0.1$ a decrease in $\Delta E_{2}$ is observed. (g-j) Ground-state energy for different values of $N_{a}$ and for positive $U$. For smaller values of $U(U \rightarrow 0) \Delta E_{2}$ becomes smaller. But just as in the $U<0$ case, for larger values of $N_{a}$, even for very small values of $U$, there is still a $\Phi_{0} / 2$ periodicity. It should be noted that in all cases, as $N_{a} \rightarrow \infty$, all oscillations vanish, $\Delta E_{1,2} \rightarrow 0$. 
5b). But for $U \rightarrow 0, U \times N_{a} \rightarrow 0 ; \Delta E_{1}=2 \pi^{2} / N_{a}^{2}$ and $\Delta E_{2}$ $=0$. The plots of energy versus flux behavior of the system for positive $U$ are shown explicitly in Figs. $5 \mathrm{~g}-5 \mathrm{j}$.

With the new state found in our work, an $h c / 2 e$ periodicity of the ground-state energy appears even for positive $U$. This branch vanishes gradually as $U \rightarrow 0$. It is not possible to find this periodicity with the Lieb-Wu solution.

\section{B. Comparison with other theories}

The energy oscillations with the $h c / 2 e$ periodicity were calculated in the strongly correlated electron models, including the Hubbard model, in a number of papers. ${ }^{11-17}$ In some papers ${ }^{18-21}$ the Hubbard model was examined by using the Lieb and Wu solution. ${ }^{9}$ The oscillations with the $h c / 2 e$ periodicity for negative $U$ can be found by starting directly from the original solution presented by Lieb and $\mathrm{Wu}$, since the new state found in our work does not become the minimum energy state. But for positive $U$, new states should be included to obtain the correct $h c / 2 e$ periodicity. The Lieb and $\mathrm{Wu}$ solution does not lead to the $h c / 2 e$ periodicity for positive $U$.

1) Why Lieb-Wu is incomplete:

Let us consider the Lieb-Wu equations (with no magnetic flux $\Phi)$

$$
\begin{aligned}
& \exp \left(i N_{a} k_{1}\right)=\frac{\sin k_{1}-\sin k_{2}+i U / 2}{\sin k_{1}-\sin k_{2}-i U / 2}, \\
& \exp \left(i N_{a} k_{2}\right)=\frac{\sin k_{2}-\sin k_{1}+i U / 2}{\sin k_{2}-\sin k_{1}-i U / 2} .
\end{aligned}
$$

Dividing the first equation by the second, with $k_{1}+k_{2}=Q$ and $k_{1}-k_{2}=2 \kappa$, we obtain

$$
\exp \left(2 i N_{a} \kappa\right)=\left(\frac{2 \sin \kappa \cos (Q / 2+\alpha)+i U / 2}{2 \sin \kappa \cos (Q / 2+\alpha)-i U / 2}\right)^{2}
$$

The energy equation is

$$
E=-2\left(\cos k_{1}+\cos k_{2}\right)=-4 \cos (Q / 2) \cos \kappa,
$$

and the new eigenvalue found by us is

$$
E=-4 \cos (Q / 2) \cos \left(\pi / N_{a}\right) .
$$

Therefore, $\kappa$ should be equal to $\pi / N_{a}$ in Eq. (27). According to Eq. (26) it is obvious that this is possible only if $U=0$. The Lieb-Wu solution does not give this result for all $U$ except $U=0$.

In the original paper of Lieb and $\mathrm{Wu}^{9}$ it is explicitly stated that the momenta $k_{j}$ should be unequal, which means that both $I_{1}-I_{2}$ and $I_{1}+I_{2}$ cannot be equal to zero $\left(I_{1}\right.$ and $I_{2}$ are integers in the original paper of Lieb and $\mathrm{Wu} .{ }^{9}$ This is also the case in our procedures. In terms of our approach, $\kappa=0$ should be excluded from the solution set. But in some papers ${ }^{14} k_{1}$ is assumed to be equal to $k_{2}$, so that $\kappa=0$ and a $\Phi_{0} / 2$ periodicity is obtained by accident.

\section{CONTRACTION MODEL}

\section{A. Physical background}

In the investigation of unusual electronic properties of metal-oxide compounds it was proposed ${ }^{7,8,22}$ that the new features in the electronic band conduction in oxide metals should be included. The first one is the possibility that "intrinsic-hole" rather than intrinsic-electron carriers may play a role. The second one is that, provided intrinsic holes are at work, one-particle picture of the electronic transport is not fully adequate, because the interaction between holes (repulsive or attractive) must be included, and because the fact that the hopping of holes in itself cannot be considered as constant in amplitude and is strongly dependent upon site occupation.

Normally, two oxygen atoms have a strong tendency to make covalent bonding, which results in the formation of an oxygen molecule, $\mathrm{O}_{2}$. However, in a proper chemical surrounding, this may not happen if the nearest neighbor atoms are not too close to each other. In this case the other scenario, which is reminiscent of metallic oxygen, applies. We can assume that this is just what happens in the metal-oxide superconductors. In the $\mathrm{CuO}_{2}$ plane of the latter, due to large ionic radii of oxygen, the oxygen orbitals overlap each other almost as strongly as the near site oxygen and copper orbitals do. The $\mathrm{O}_{2}$ molecules therefore are not formed, and the electrons derived from the $p^{6}$ shell are the conducting electrons. The charge carriers are holes in the $p^{6}$ shell, which propagate from one oxygen anion to the next nearest one by hopping. Because of the contraction of the $p$ orbital of oxygen as a result of occupation by a hole, hole hopping between nearest-neighbor sites $(i, j)$ is dependent on the opposite-spin hole occupation number. In the second quantization representation it was suggested to consider the hopping matrix element $t_{i j}$ as an operator which depends on the occupation number operators $n_{i}$ and $n_{j}$ at the atomic sites $R_{i}$ and $R_{j}$. There are three independent matrix elements, $t_{0}, t_{1}$, and $t_{2}$ (Refs. 23 and 26), which in the case of two oxygen anions correspond to the following, charge transfer reactions:

$$
\begin{array}{ll}
t_{0}: & \mathrm{O}_{i}^{-}+\mathrm{O}_{j}^{2-} \rightarrow \mathrm{O}_{i}^{2-}+\mathrm{O}_{j}^{-} \\
t_{1}: & \mathrm{O}_{i}+\mathrm{O}_{j}^{2-} \rightarrow \mathrm{O}_{i}^{2-}+\mathrm{O}_{j}, \\
t_{2} ; & \mathrm{O}_{i}+\mathrm{O}_{j}^{-} \rightarrow \mathrm{O}_{i}^{-}+\mathrm{O}_{j},
\end{array}
$$

which result in

$$
\begin{aligned}
t_{i j}= & t_{0}\left(1-n_{i,-\sigma}\right)\left(1-n_{j,-\sigma}\right)+t_{1}\left[n_{i,-\sigma}\left(1-n_{j,-\sigma}\right)\right. \\
& \left.+n_{j,-\sigma}\left(1-n_{i,-\sigma}\right)\right]+t_{2} n_{i,-\sigma} n_{j,-\sigma} .
\end{aligned}
$$

The occupation dependence of the hopping can be represented in another form:

$$
t_{i j}=-t+V n_{i,-\sigma} n_{j,-\sigma}+W\left(n_{i,-\sigma}+n_{j,-\sigma}\right),
$$

where from Eq. (30) we obtain

$$
t=-t_{0}, \quad V=t_{0}-2 t_{1}+t_{2}, \quad W=t_{1}-t_{0} .
$$

Hence, the $1 D$ version of the interacting holes in an anion network can be represented by the following Hamiltonian, which includes the on-site interaction term $U$ : 


$$
\begin{aligned}
H= & -\sum_{j \sigma} c_{j \sigma}^{+} c_{j+1, \sigma} \exp (i \alpha)+\text { H.c. }+U \sum_{j} n_{j \uparrow} n_{j \downarrow} \\
& +\sum_{j, \sigma} c_{j \sigma}^{+} c_{j+1, \sigma}\left[V n_{j,-\sigma} n_{j+1,-\sigma}+W\left(n_{j,-\sigma}\right.\right. \\
& \left.\left.+n_{j+1,-\sigma}\right)\right] \exp (i \alpha)+\text { H.c. }
\end{aligned}
$$

The effect of the coupling term $\mathrm{W}$ has been considered in great detail in the paper of Hirsch and Marsiglio, ${ }^{7}$ as well as by Kulik et al..$^{8,25}$

\section{B. Bound state of two electrons}

As before, we use the wave function for two electrons, one with spin up and the other with spin down,

$$
|\Psi\rangle=\sum_{x_{1}, x_{2}} f\left(x_{1}, x_{2}\right) c_{x_{1} \downarrow}^{+} c_{x_{2} \uparrow}^{+}|0\rangle .
$$

In momentum space the eigenvalue equation $H|\Psi\rangle=E|\Psi\rangle$ gives

$$
\left[\begin{array}{cc}
1-U S_{0}(E)-W S_{1}(E) & -W S_{0}(E) \\
U S_{1}(E)+W S_{2}(E) & -1+W S_{1}(E)
\end{array}\right] \times\left[\begin{array}{c}
F_{0}(Q) \\
F_{1}(Q)
\end{array}\right]=0,
$$

where

$$
\frac{1}{N_{a}} \sum_{K}\left(\varepsilon_{K_{1}-K+\alpha}+\varepsilon_{K_{2}+K+\alpha}\right)^{n} f_{K_{1}-K, K_{2}+K} \equiv F_{n}(Q),
$$

$n=0,1$, and

$$
\frac{1}{N_{a}} \sum_{p} \frac{\left(\varepsilon_{K_{1}-p+\alpha}+\varepsilon_{K_{2}+p+\alpha}\right)^{n}}{E+\left(\varepsilon_{K_{1}-p+\alpha}+\varepsilon_{K_{2}+p+\alpha}\right)} \equiv S_{n}(E)
$$

$n=0,1,2 ; \varepsilon_{k}=2 \cos k$. Hence, either the determinant of the first matrix is equal to zero or both terms of the vector are zero.

For two electrons $V$ does not show up. The effect of $V$ in the weak-coupling regime was considered previously. ${ }^{8}$

In the case $F_{0}=F_{1}=0$ the energy eigenvalue of the system becomes

$$
\begin{aligned}
E & =-2 \cos (q+\alpha)-2 \cos (Q-q+\alpha) \\
& =-4 \cos (Q / 2-q) \cos \beta .
\end{aligned}
$$

It is possible to have both $F_{0}$ and $F_{1}$ equal to zero, while all $f$ 's are not individually equal to zero only if for two different values of $q, 2 \cos (q+\alpha)+2 \cos (Q-q+\alpha)$ are coinciding.

For the other case, i.e., when determinant of the first matrix in Eq. (35) is equal to zero, the transcendental equation is found as follows:

$$
\frac{(W-1)^{2}}{U+W(W-2) E}=S_{0}(E) \text {. }
$$

The plot of $S_{0}(E)$ is presented in Fig. 6. Equation (39) can be solved numerically, which is done to test our results. If we set $W=0$ in the last equation, we immediately obtain the

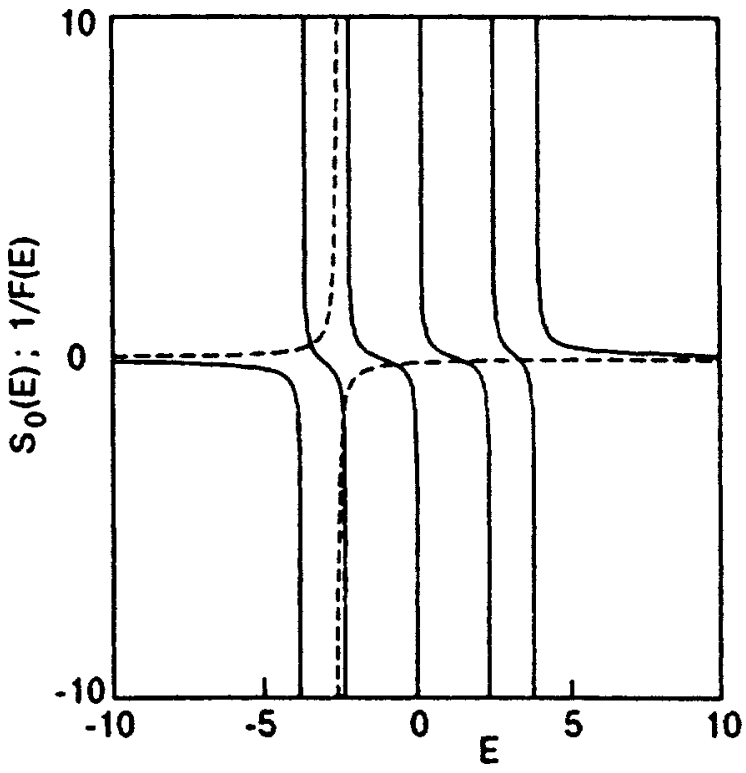

FIG. 6. Plot of the transcendental equation for the contraction model. The intersection points of $S_{0}(E)$ (solid line) with $1 / F(E)$ (dashed line) give the energy eigenvalues. Here $N_{a}=10, \Phi=\Phi_{0} / 2, n=9, U=-2$, and $W=1.5$.

result of the $1 D$ Hubbard model discussed in Sec. 2. With similar calculations as in the previous sections, the minimum energy corresponding to Eq. (39) is found as

$$
E=-\left(\cos k_{1}+\cos k_{2}\right)=-4 \cos x \cos \beta,
$$

where $x$ is determined by

$$
\tan \frac{N_{a} x}{2}=-\sigma\left(\frac{4(W-1)^{2} \sin x \cos \beta}{U-4 W(W-2) \cos x \cos \beta}\right)^{\sigma} .
$$

Here $\sigma=+1$ or -1 for odd or even values of $n$. In the hatched region in Fig. 7 for odd values of $n$ the expression

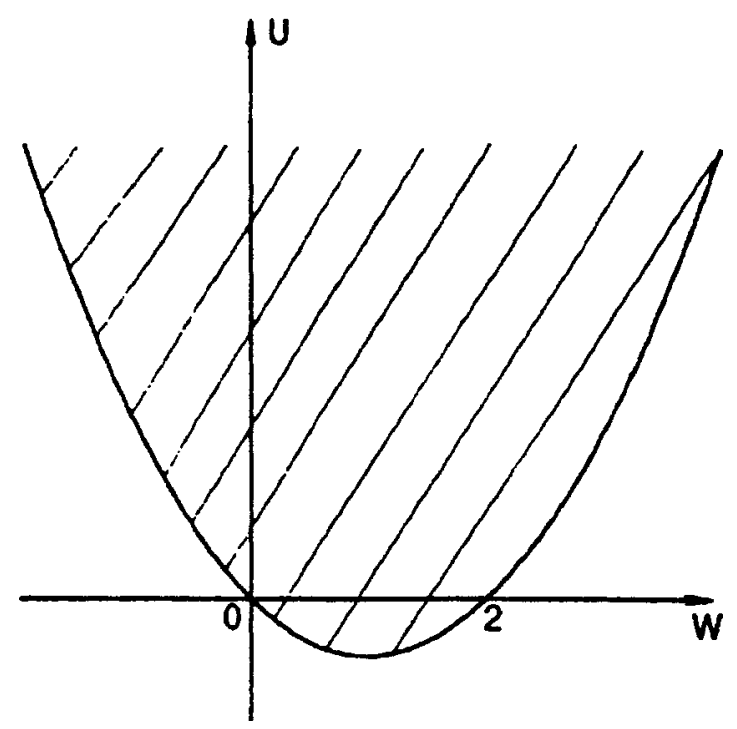

FIG. 7. Phase space for bound states of two electrons. The hatched region corresponds to the free propagating states and the nonhatched region corresponds to the bound states of two electrons within the contraction model. The solid line corresponds to the equation $U=-W(W-2) E_{1}$, where $E_{1}$ $=-4 \cos \beta$ for even $n$ and $E_{1}=-4 \cos \beta \cos \left(\pi / N_{a}\right)$ for odd $n$. 
TABLE II. Minimum energy for different values of $U$.

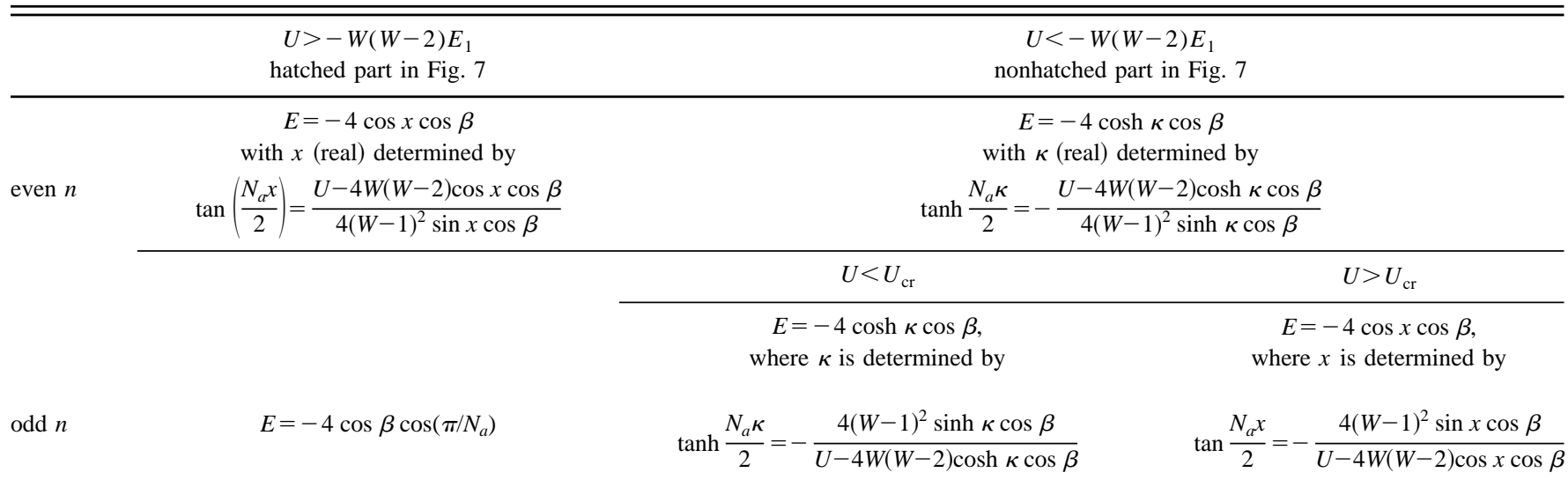

$$
E=-4 \cos \left(\pi / N_{a}\right) \cos \beta
$$

gives the minimum energy value. The curve in Fig. 7 corresponds to $U=-W(W-2) E_{1}$, where $E_{1}=-4 \cos \beta$ for even $n$ and $E_{1}=-4 \cos \beta \cos \left(\pi / N_{a}\right)$ for odd $n$. The resulting values of the ground-state energy for different values of $U$ and $W$ are summarized in Table II.

Here $U_{\mathrm{cr}}$ is found in a similar way to that of the Hubbard model. The energy-versus-flux dependence for two electrons in the contraction model is shown in Fig. 8.

The amplitudes of the energy oscillations in the $N_{a} \gg 1$ limit are found as follows:

(i) For the nonhatched region below the curve (the bound states) and $U<U_{\mathrm{cr}}\left(U<U_{\mathrm{cr}}<-W(W-2) E_{1}\right)$ :

$\Delta E_{1}=\Delta E_{2}=\Delta E$

$\approx \frac{\left(2 \pi^{2} / N_{a}^{2}\right)(W-1)^{4}}{\left\{U^{2} W^{2}(W-2)^{2}+\left(2 W^{2}-4 W+1\right)\left[16(W-1)^{4}+U^{2}\right]\right\}^{1 / 2}}$.

Hence there is a $\Phi_{0} / 2$ periodicity. The branch corresponding to the expression in Eq. (42) for odd $n$ does not become the minimum energy; it is shown as a dashed line in Fig. 8a. As $U \rightarrow U_{\text {cr }}$ from below, the branch which is marked as 2 in Fig. $8 \mathrm{c}$ fades away from being the minimum energy. Eventually, at $U=U_{\text {cr }}$ there is no more $\Phi_{0} / 2$ periodicity. For very large $N_{a}\left(N_{a} \rightarrow \infty\right), U_{\mathrm{cr}} \rightarrow 4 W(W-2)$. It is interesting that in this very large $N_{a}$ limit $E_{1} \rightarrow-4$, so that the curve in Fig. 7 corresponds to $U=4 W(W-2) \sim U_{\mathrm{cr}}$. Hence, for very large $N_{a}$, any $U$ which satisfies $U<4 W(W-2)$ is less than $U_{\mathrm{cr}}$; therefore, almost always there is a $\Phi_{0} / 2$ periodicity in the nonhatched region in Fig. 7.

(ii) For the shaded region above the curve in Fig. 7 the expression in Eq. (42) becomes the minimum energy of the system. This branch is shown as the dashed line in Fig. 8a. The corresponding amplitudes are

$$
\begin{aligned}
& \Delta E_{1} \approx\left(2 \pi^{2} / N_{a}^{2}\right)(1-\lambda)^{2}, \\
& \Delta E_{2} \approx\left(2 \pi^{2} / N_{a}^{2}\right) \lambda^{2},
\end{aligned}
$$

where

$$
\lambda=\frac{1}{2}\left(\frac{(U-4 W(W-2)) N_{a}}{8(W-1)^{2}+N_{a}[U-4 W(W-2)]}\right)^{2} .
$$

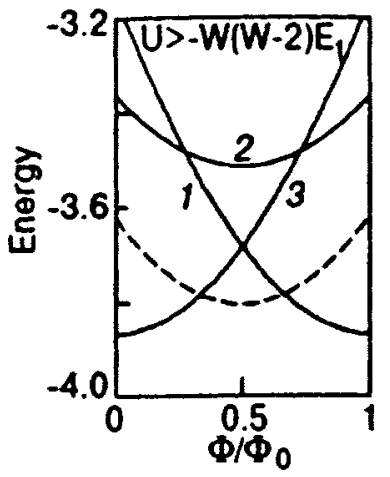

c

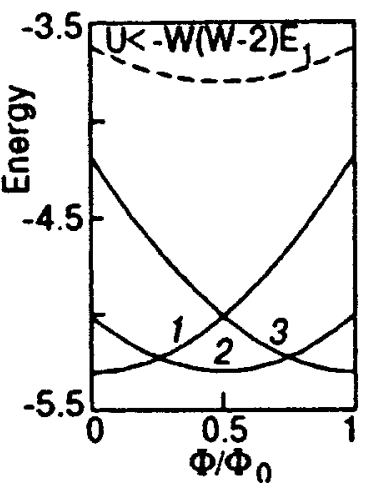

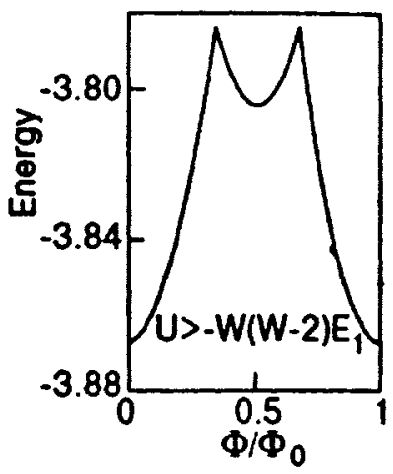

d

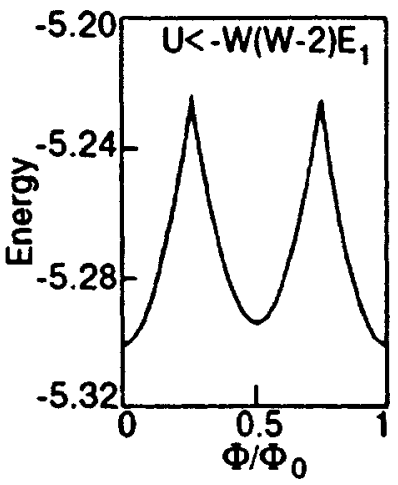

FIG. 8. Energy versus flux for two electrons in the contraction mechanism. Note the resemblance of this figure to Fig. 3. Here instead of $U>0$ there is $U>-W(W-2) E_{1}$; similarly for $U<0$ there is the $U<-4 W(W-2)$ criterion. In (a) the solid curves correspond to the expression (40) and the dashed curve corresponds to the expression (42). Just like for $U>0$ in the Hubbard model, in the contraction model for $U>-W(W-2) E_{1}$ the dashed curve becomes the minimal energy of the model. (b) The same as (a) to show the behavior of the system more clearly. In (a) and (b) $N_{a}=10, U$ $=-2, W=1.5$. In (c) $U<-W(W-2) E_{1}$, just as in the Hubbard model for $U<0$, the solution corresponding to Eq. (42) does not take place as the minimum energy of the model. The solid curves $1-3$ correspond to Eq. (40) and the dashed curve corresponds to Eq. (42). (d) is the same as (c) to show the behavior more clearly. In (c) and (d) $N_{a}=10, U=2$, and $W=-1$. 
For $(U-4 W(W-2)) N_{a} \rightarrow \infty, \quad \Delta E_{1}=\Delta E_{2}=1 / 4\left(2 \pi^{2} / N_{a}^{2}\right)$. But for $(U-4 W(W-2)) N_{a} \rightarrow 0, \Delta E_{1}=2 \pi^{2} / N_{a}^{2}$, and $\Delta E_{2}$ $=0$.

All results found here and in the previous section for the Hubbard model are in close correlation. In the Hubbard model and the contraction model two different types of solutions were found. For the Hubbard model a new type of solution gives the $\Phi_{0} / 2$ periodicity for $U>0$, which is absent in the Lieb-Wu solution, while in the contraction model this type of solution gives the $\Phi_{0} / 2$ periodicity for $U>-W(W$ $-2) E_{1}$. In the Hubbard model for $U<0$ can be larger or smaller than $E_{0}$, depending on whether $U$ is larger or smaller than a critical value $U_{\mathrm{cr}}$. Similarly for the contraction model for $U<-W(W-2) E_{1}, E$ can be larger or smaller than $E_{0}$, depending on whether $U$ is larger or smaller than $U_{\mathrm{cr}}$. For Hubbard model $U_{\mathrm{cr}}$ becomes zero for very large $N_{a}$, for contraction model it becomes $4 W(W$ $-2)$. In all these inequalities one can get Hubbard model type relations setting $W=0$ in contraction model relations.

\section{CONCLUSIONS}

In the one-dimensional Hubbard model and the contraction model for two electrons, the periodicity of ground-state energy with respect to flux is $h c / 2 e$. Our study shows that the solution for a one-dimensional Hubbard model by Lieb and $\mathrm{Wu}^{9}$ in 1968 is not complete, at least for two electrons. For positive on-site interaction new states found by us correspond to the ground-state energy. Hence, they play an important role for correct behavior of the ground-state energy of the system. Generalizing the current results to more than two electrons will be the task of a future work. It is very likely that for more than two electrons new states, which cannot be determined by the Lieb and $\mathrm{Wu}$ results, will be found. The model for the ground-state energy of contraction has a $h c / 2 e$ periodicity also. But it is not easy to speak about superconductivity very clearly. For some range of the values of $U$ and $W$ it is likely that this model results in superconductivity. To show that this model serves as a model for superconductivity, other probing methods should be used.

This work was supported in part by the Scientific and Technical Research Council of Turkey TÜBITAK.
*Permanent address: B. Verkin Institute for Low Temperature Physics and Engineering, Nat. Acad. Sci. of Ukraine, 47, Lenin Ave., 310164 Kharkov, Ukraine

**E-mail: kulik@fen.bilkent.edu.tr

${ }^{1}$ E. Dagotto, Rev. Mod. Phys. 66, 763 (1994).

${ }^{2}$ P. W. Anderson, Science 235, 1196 (1987).

${ }^{3}$ G. Kotliar, Adv. Phys. 44, 187 (1995).

${ }^{4}$ D. Pines, Tr. J. Phys. 20, 535 (1996).

${ }^{5}$ D. J. Scalapino, Phys. Rep. 250, 329 (1995).

${ }^{6}$ J. R. Schrieffer, X. G. Wen, and S. C. Zhang, Phys. Rev. B 39, 11663 (1989).

${ }^{7}$ J. E. Hirsch and F. Marsiglio, Phys. Rev. B 39, 11515 (1989).

${ }^{8}$ I. O. Kulik, Sverhprovodimost': Fiz., Khim., Tekh. 2, 175 (1989) [Sov. Supercond.: Phys., Chem., Technol. 2, 201 (1989)].

${ }^{9}$ E. H. Lieb and F. Y. Wu, Phys. Rev. Lett. 20, 1445 (1968).

${ }^{10}$ I. O. Kulik, Tr. J. Phys. 20, 627 (1996).

${ }^{11}$ A. Sudbo, C. M. Varma, T. Giamarchi, E. B. Stechel, and R. T. Scalettar, Phys. Rev. Lett. 70, 978 (1993).

${ }^{12}$ R. M. Fye, M. J. Martins, and D. J. Scalapino, Phys. Rev. B 44, 6909 (1991).

${ }^{13}$ A. A. Zvyagin, Zh. Éksp. Teor. Fiz. 103, 307 (1993) [JETP 76, 167 (1993)].

${ }^{14}$ F. V. Kusmartsev, J. F. Weisz, R. Kishore, and M. Takahashi, Phys. Rev. B 49, 16234 (1994).

${ }^{15}$ A. Ferretti, I. O. Kulik, and A. Lami, Physica C 185, 1649 (1991).

${ }^{16}$ J. F. Weisz, R. Kishore, and F. V. Kusmartsev, Phys. Rev. B 49, 8126 (1994).

${ }^{17}$ A. Ferretti, I. O. Kulik, and A. Lami, Phys. Rev. B 45, 5486 (1992).

${ }^{18}$ A. A. Zvyagin and I. V. Krive, Zh. Éksp. Teor. Fiz. 102, 1376 (1992) [Sov. Phys. JETP 75, 745 (1992)].

${ }^{19}$ A. A. Zvyagin, Fiz. Tverd. Tela 32, 1546 (1990) [Sov. Phys. Solid State 32, 905 (1990)].

${ }^{20}$ A. A. Zvyagin, Fiz. Nizk. Temp. 17, 1436 (1991) [Sov. J. Low Temp. Phys. 17, 779 (1991)].

${ }^{21}$ Kong-Ju-Block Lee and P. Schlottmann, Phys. Rev. B 38, 11566 (1988).

${ }^{22}$ J. E. Hirsch, Phys. Lett. A 134, 451 (1989).

${ }^{23}$ I. O. Kulik, Contraction Mechanism for Pairing Interaction in Oxides and Hydrides, in Progr. in High Temperature Physics, Vol. 25, R. Nicholsky (Ed.), World Scientific, Singapore (1990).

${ }^{24}$ I. O. Kulik, Fiz. Nizk. Temp. 17, 1195 (1991) [Sov. J. Low Temp. Phys. 17, $628(1991)]$.

${ }^{25}$ A. Ferretti, I. O. Kulik, and A. Lami, Phys. Rev. B 47, 12235 (1993).

${ }^{26}$ H. Q. Lin and J. E. Hirsch, Phys. Rev. B 52, 16155 (1995).

${ }^{27}$ I. O. Kulik and A. G. Pedan, Zh. Éksp. Theor. Phys. 79, 1469 (1980) [Sov. Phys. JETP 52, 742 (1980)].

${ }^{28}$ I. O. Kulik, Int. J. Mod. Phys. B1, 851 (1988).

This article was published in English in the original Russian journal. It was edited by S. J. Amoretty. 